\title{
Multiway principal component analysis (MPCA) contributions for structural damage localization
}

\author{
Magda Ruiz ${ }^{1}$, Luis Eduardo Mujica ${ }^{1}$, Julián Sierra ${ }^{2}$, Francesc Pozo ${ }^{1}$ and José Rodellar ${ }^{1}$
}

\begin{abstract}
In this paper, a novel methodology for damage localization is introduced. The approach is based on a multiactuator system. This means that the system itself has the ability of both exciting the specimen and measuring its response at different points in a pitch-catch mode. Once one of its actuators excites the specimen, the damage affects the normal travel of the guided wave, and this change is mainly detected by sensors in the direct route to the excitation point. In previous works by the authors, it can been observed the progression using data-driven statistical models (multivariable analysis based on principal component analysis, PCA) of all recorded signals to determine whether the damage is present. However, the main contribution of this paper is the demonstration of the possibility of localizing damages by analyzing the contribution of each sensor to this index which have detected it ( $T^{2}$-statistic and $Q$-statistic). The proposed methodology has been applied and validated on an aircraft turbine blade. The results indicate that the presented methodology is able to accurately locate damages, analyzing the record signals from all actuation phases, giving a unique and reliable region.
\end{abstract}

\section{Keywords}

principal component analysis, contribution analysis, damage localization

\section{Introduction}

The principal challenge in structural health monitoring (SHM) is to detect damages in an incipient stage guaranteeing the integrity of the structure and hence, increasing the security, reducing costs of maintenance and repair. In structures, a damage is defined as a "change in material and / or geometrical properties introduced in the structure that affects negatively the current and future performance of it" (1). Nowadays, new technologies are being constantly developed and incorporated in order to improve the efficiency and accuracy in the monitoring methods. Many of these SHM approaches have been developed by analyzing the structural dynamic response signals captured by several sensors that are permanently integrated to the structure collecting information throughout its service life. The approaches are based on the premise that referencing baseline signals (gathered from a structure supposed to be pristine), the damage can be recognized. Those approaches based on changes in global dynamic properties including eigen-frequency, mode shape and curvature, strain energy, and damping properties are less sensitive to emerging damages since they are local events which would no affect structural global responses. Electromechanical impedance, displacement, strain and acoustic emission are features that can be used to detect local damages, however, the guided wave-based approaches present advantages including capability of propagation over long distances and high sensitivity to abnormalities near to the wave propagation path. Elastic waves are induced into the structure and any discontinuity in the propagation medium (damage) can be identified by examining the scattered wave signals (2).
The most promising techniques under development involve the use of active piezoelectric multiactuator systems that sequentially excite in the same way (known excitation) different points and, record the vibration characteristics (or guided elastic waves) of the structure in several locations. All this information is used to make estimates regarding the health of the structure. These kind of data have been shown to be highly sensitive for damage detection and provide several advantages over passive approaches such as strain monitoring and acoustic emission monitoring.

All these monitoring systems manage a great quantity of information and have large volume of historical data stored in databases. Exploitation of these data is a critical component in the successful operation of monitoring over the long term, however, until the last two decades, nothing has been done with them, due to the nature of these data. This amount of data is enormous and often highly correlated. To make the most of these data, a database must be able to deal effectively with all these difficulties. In this way, researchers

${ }^{1}$ Control, Dynamics and Applications (CoDAlab), Departament de Matemàtiques, Escola d'Enginyeria de Barcelona Est (EEBE), Universitat Politècnica de Catalunya (UPC), Eduard Maristany, 6-12, 08930 Sant Adrià de Besòs, Barcelona, Spain.

${ }^{2}$ Grupo de Investigación en Ingeniería Aeroespacial. Facultad de Ingeniería Aeronáutica. Universidad Pontificia Bolivariana. Medellín, Colombia.

\section{Corresponding author:}

Magda Ruiz, Departament de Matemàtiques, Escola d'Enginyeria de Barcelona Est (EEBE), Universitat Politècnica de Catalunya (UPC), Campus Diagonal-Besòs, Eduard Maristany, 6-12, 08930 Sant Adrià de Besòs, Barcelona, Spain

Email: magda.ruiz@upc.edu 
like McGregor, Nomikos, Kourti and Wold, among others, started focusing on developing statistical data-driven models by using latent variable methods mainly in the field of chemometrics (3), (4) and (5). One of this latent methods is named principal component analysis (PCA), which is a good tool for data compression and information extraction which find combinations of variables or factors that describe major trends in a data set.

In general, to detect, discriminate and identify defects in structures, it is best to create a good statistical model to recognize characteristics or patterns of the acquired signals related to the defects. In an initial state, with the pristine structure, the actuators are excited with a known signal and the dynamic response (guided waves) is captured throughout the structure by means of sensors and later, is processed in order to get a model that finally is stored. When the structure begins to be used, all actuators and sensors are continuously exciting and recording information. If defects that alter the stiffness, mass or energy dissipation properties of the structure appear, the dynamic response changes and a warning signal has to be marked.

In previous works by the authors, it can been observed the progression using data-driven statistical models in SHM, since the use of PCA and both $T^{2}$-statistic and $Q$ statistic indices as elements for damage detection (6) to the most recent wherein partial least squares (PLS) is used as regression tool to localize impacts (7). Besides, there are works where new extensions have been implemented: For instance, the projections to principal components are considered as random variables by nature and, they are used to implement statistic inference for damage detection in the cases where the projections are not sensitive to damage by themselves (8).

The likely presence of damage can be recognized quite simply on the basis of anomalies in the dynamic response. However, giving more precise information about the position and nature of the damage is more complicated. Its complexity depends on the structure, the type of sensors and their distribution. After detecting the damage, the next challenge for SHM is to determine its localization (9).

Several methods have been proposed in the literature to localize damage based on guided waves. A number of methods rely on modal parameters -e.g. (10)-, other on operational shapes retrieved from frequency response functions (FRF) -e.g. (11)-. From the point of view of time-domain response, the reported methods are mainly based on the definition of the arrival time of the reflected elastic waves, specifically the time-of-flight of the signals (TOF). The traditional methods to calculate the TOF of the propagating signals mainly include the thresholding value, correlation coefficient (12), Hilbert envelope (13)(14), wavelet transform (15), Wigner Ville distribution (16), optimization of nonlinear equations (18), and short time Fourier transformation methods (19), among others.

A comparison of several damage localization techniques in a structure with constant thickness can be found in (20). These techniques are based on variations of TOF method, RAPID-method, energy of arrival and total product method. In the case of isotropic structures with smoothly varying thickness, an approach based on the fundamental symmetric wave mode is presented in (21). On the other hand, damage localization in a flat anisotropic structures has been demonstrated in (22)(23).

More recently, the stochastic dynamic damage locating vector (SDDLV) method based on both a finite element (FE) model of the structure and modal parameters estimated from output-only measurements in the damage is used to localize damages in (26) and (27). A vibration based statistical time series method that is capable of damage detection, localization, and magnitude estimation within a unified stochastic framework is presented in (28)(29). The method is based on the novel extended class of vector-dependent functionally pooled (VFP) models and proper statistical decision making schemes. A time-reversal technique for the estimation of a damage location and sizing in a composite plate was reported in (30). It relies on the analytically based numerical simulation of the PWAS induced re-emitted guided wave signals measured with laser Doppler vibrometry at a sparse set of points on the specimen surface.

In general, an accurate interpretation of captured guided wave signals is critical for damage localization strategies, since the captured signals are usually complex due to the multiple wave modes, wave dispersion, boundary reflection, noise, and so on. To avoid direct interpretation of signals and the aforementioned effects, techniques based on pattern recognition have been attracting attention. For instance, probability-based diagnostic imaging (PDI) has been studied intensively by many researchers. Among them, in (31) it is proposed an algorithm based on weight-compensated PDI to improve the ability of damage localization.

As previously mentioned, keeping the same idea of pattern recognition for damage detection and discrimination, the well-known PCA tool has been recurrently used by the authors of this work. Now, the main contribution of this paper is the demonstration of the possibility of localizing damages by analyzing the contribution of each sensors to the indices that have detected the damage. To achieve this, only measurements from PZT sensors distributed over the structure are used. No previous information about the damage localization is required. At the end, the methodology will provide a possible region where the damage can be located

The paper is organized in the following way: In the second section, after this brief introduction, a theoretical review about multiway principal component analysis (MPCA) is presented, this section is focused on the explanation of the arrangement of the collected data according to the nature of the experimentation, the re-organization of these data to apply PCA, standard PCA and its damage indices: $T^{2}$ statistic and $Q$-statistic (also known as control charts). Section 3 is devoted to the mathematical background related to the analysis of the contribution (also known as contribution plots) focusing on the formal definition and the formulation for each damage indicator. Next, the general methodology proposed in this paper and the main contribution are detailed and the different phases are detailed: Damage detection, contribution analysis and damage localization. Following, the experimental setup is described: the used specimen, the multiactuator system, excitation signal and experimental design. Finally, results are analyzed and discussed drawing the main conclusions and contributions of the work. 


\section{Multiway principal component analysis (MPCA)}

MPCA is a straightforward extension of conventional PCA to handle data in multi-dimensional arrays. A typical 2D data matrix can be considered as a two-way array, with experiments and variables (or discretization instant times) forming the two different ways. In some applications, it is necessary to extend this scheme to multiway arrays, for instance if in different experimental trials, several sensors are measuring at different time instants. MPCA is equivalent to performing ordinary PCA to an unfolded version of the original multiway array.

\section{Organization of the recorded data}

Let us address the analysis by measuring just one sensor at a given number of discretization instants. This measurement is repeated several times (experimental trials), considering that each measurement is an individual experiment in the data set (i.e. dynamical response, e.g. stress, strain, load, voltage, pressure, etc.). The collected data are arranged as follows:

$$
\begin{aligned}
\mathbf{X}= & \left(\begin{array}{cccccc}
x_{11} & x_{12} & \cdots & x_{1 k} & \cdots & x_{1 K} \\
\vdots & \vdots & \ddots & \vdots & \ddots & \vdots \\
x_{i 1} & x_{i 2} & \cdots & x_{i k} & \cdots & x_{i K} \\
\vdots & \vdots & \ddots & \vdots & \ddots & \vdots \\
x_{I 1} & x_{I 2} & \cdots & x_{I k} & \cdots & x_{I K}
\end{array}\right) \\
= & \left(\begin{array}{c}
x_{1}^{T} \\
\vdots \\
x_{i}^{T} \\
\vdots \\
x_{I}^{T}
\end{array}\right)=\left(v_{1}\left|v_{2}\right| \cdots\left|v_{k}\right| \cdots \mid v_{K}\right)
\end{aligned}
$$

This matrix $\mathbf{X} \in \mathcal{M}_{I \times K}(\mathbb{R})$-where $\mathcal{M}_{I \times K}(\mathbb{R})$ is the vector space of $I \times K$ matrices over $\mathbb{R}$ - contains information from $K \in \mathbb{N}$ time instants and $I \in \mathbb{N}$ experimental trials. Each row vector $\left(x_{i}^{T}\right)$ represents measurements from the sensor at a specific $i$ th trial. In the same way, each column vector $\left(v_{k}\right)$ represents measurements at the specific $k$ th time instants in the whole set of experiment trials.

Considering that a multiactuator system is used, $J \in \mathbb{N}$ sensors are continuously gathering data by each trial experiment. The collected data are arranged as follows:

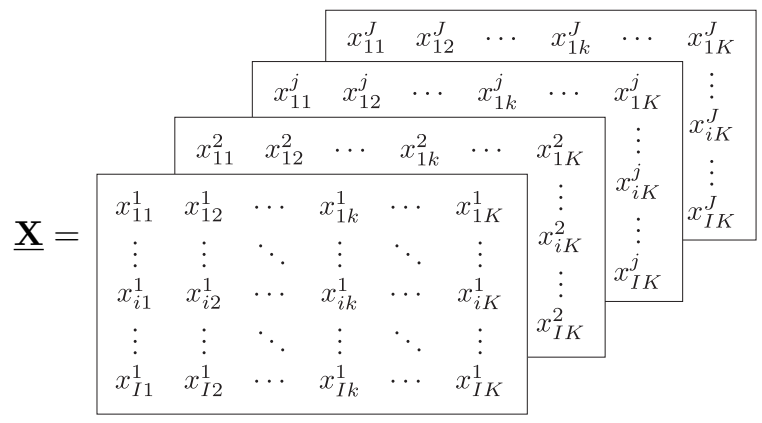

This $\underline{\mathbf{X}} \in \mathcal{M}_{I \times K \times J}(\mathbb{R})$-where $\mathcal{M}_{I \times K \times J}(\mathbb{R})$ is the vector space of $I \times K \times J$ matrices over $\mathbb{R}$ - contains information from $K$ discretization instant times, $I$ experimental trials and $J$ sensors. Each row vector $\left(x_{i}^{j}\right)^{T}$ represents the measurements from the $j$ th sensor at a specific $i$ th trial.

\section{Unfolding}

To apply PCA to a three-way data matrix $\underline{\mathbf{X}}$ as in equation (2), it has to be unfolded into a two-dimensional data matrix X (32) (33). According to (34), there are six possible ways of unfolding this three-way data $\underline{\mathbf{X}}$ matrix, as indicated in Table 1 .

\begin{tabular}{ccc}
\hline Type & Dimension & Direction \\
\hline$A$ & $I K \times J$ & sensors \\
$B$ & $J I \times K$ & time \\
$C$ & $I J \times K$ & time \\
$D$ & $I \times K J$ & experiment \\
$E$ & $I \times J K$ & experiment \\
$F$ & $J \times I K$ & sensors \\
\hline
\end{tabular}

Table 1. Different ways to unfold a three way data matrix

The structure of each type denotes the dimension of the resulting unfolded matrix. On the other hand, the direction of the unfolding type indicates the variable that remains unaltered (the other two variables are combined). Focusing on the basic PCA theory that will be explained in the next sections, unfolding types $B$ and $D$ will lead to models that are equivalent to models constructed using the $C$ and $E$ unfolded matrices, respectively. The matrix resulting of unfolding $F$ is the transpose of the matrix resulting after unfolding type $A$, therefore a PCA would simply switch the scores and loadings of the two matrices if no pre-processing is applied. Unfolding as $E$, see equation (3), produces a matrix where the rows are the signals from all sensors (from the same experiment) arranged one after the other, while in unfolding type $D$ each row is organized by each time instant for each sensors (from the same experiment). This means that all the measures of the first time instant for each sensor are located in the first group of data. The second group will consist on the measures of the second time-instant, again for all sensors, and so on. However, it is not possible to observe the signal profile. In both cases (usually called experiment-wise unfolding: type $D$ and $E$ ) the information about experiments are preserved. However, they present a problem for on-line monitoring because all the experiments must have the same number of samples. Alternatively, (35) suggests a sensor-wise unfolded for partial least square (PLS) approaches, which does not require complete experiments -see equation (4)-. Nevertheless, correlation between sensors is disregarded. In this work, as it has typically been used in SHM, unfolding type $E$-as in equation (3)- has been used due to the fact that the whole set of experiments have the same length and, besides, we want to preserve the information of the experiments to study the correlation between sensors and time-instants. 


$\mathbf{X}=$\begin{tabular}{cccccc|cccccc||cccccc||ccccc|c|}
\hline$x_{11}^{1}$ & $x_{12}^{1}$ & $\cdots$ & $x_{1 k}^{1}$ & $\cdots$ & $x_{1 K}^{1}$ & $x_{11}^{2}$ & $x_{12}^{2}$ & $\cdots$ & $x_{1 k}^{2}$ & $\cdots$ & $x_{1 K}^{2}$ & $x_{11}^{j}$ & $x_{12}^{j}$ & $\cdots$ & $x_{1 k}^{j}$ & $\cdots$ & $x_{1 K}^{j}$ & $x_{11}^{J}$ & $x_{12}^{J}$ & $\cdots$ & $x_{1 k}^{J}$ & $\cdots$ & $x_{1 K}^{J}$ \\
$\vdots$ & $\vdots$ & $\ddots$ & $\vdots$ & $\ddots$ & $\vdots$ & $\vdots$ & $\vdots$ & $\ddots$ & $\vdots$ & $\ddots$ & $\vdots$ & $\vdots$ & $\vdots$ & $\ddots$ & $\vdots$ & $\ddots$ & $\vdots$ \\
$x_{i 1}^{1}$ & $x_{i 2}^{1}$ & $\cdots$ & $x_{i k}^{1}$ & $\cdots$ & $x_{i K}^{1}$ & $x_{i 1}^{2}$ & $x_{i 2}^{2}$ & $\cdots$ & $x_{i k}^{2}$ & $\cdots$ & $x_{i K}^{2}$ & $x_{i 1}^{j}$ & $x_{i 2}^{j}$ & $\cdots$ & $x_{i k}^{j}$ & $\cdots$ & $x_{i K}^{j}$ & $x_{i 1}^{J}$ & $x_{i 2}^{J}$ & $\cdots$ & $x_{i k}^{J}$ & $\cdots$ & $x_{i K}^{J}$ \\
$\vdots$ & $\vdots$ & $\ddots$ & $\vdots$ & $\ddots$ & $\vdots$ & $\vdots$ & $\vdots$ & $\ddots$ & $\vdots$ & $\ddots$ & $\vdots$ & $\vdots$ & $\vdots$ & $\ddots$ & $\vdots$ & $\ddots$ & $\vdots$ & $\vdots$ & $\vdots$ & $\ddots$ & $\vdots$ & $\ddots$ & $\vdots$ \\
$x_{I 1}^{1}$ & $x_{I 2}^{1}$ & $\cdots$ & $x_{I k}^{1}$ & $\cdots$ & $x_{I K}^{1}$ & $x_{I 1}^{2}$ & $x_{I 2}^{2}$ & $\cdots$ & $x_{I k}^{2}$ & $\cdots$ & $x_{I K}^{2}$ & $x_{I 1}^{j}$ & $x_{I 2}^{j}$ & $\cdots$ & $x_{I k}^{j}$ & $\cdots$ & $x_{I K}^{j}$ & $x_{I 1}^{J}$ & $x_{I 2}^{J}$ & $\cdots$ & $x_{I k}^{J}$ & $\cdots$ & $x_{I K}^{J}$ \\
\hline
\end{tabular}

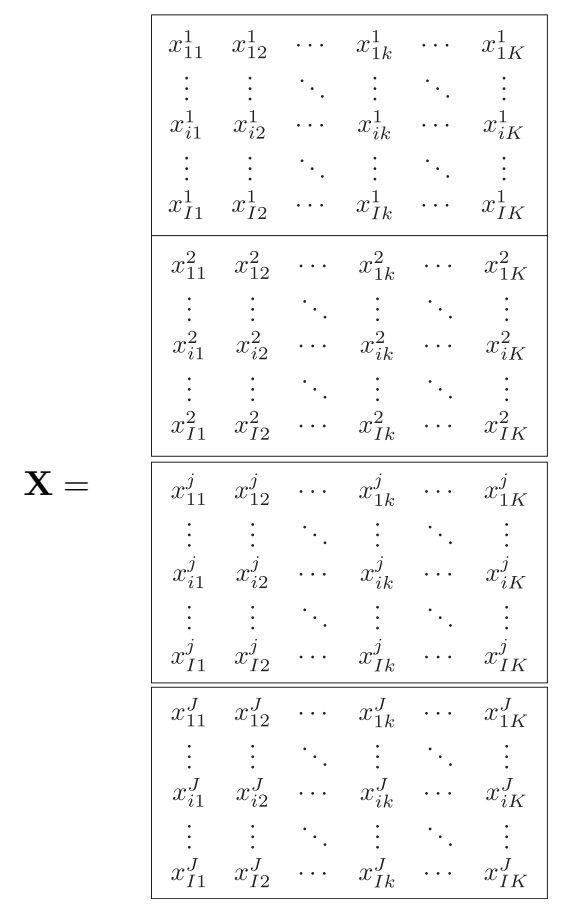

\section{Principal component analysis (PCA)}

The matrix obtained after unfolding the matrix in equation (2) as the one in equation (3), $\mathbf{X} \in \mathcal{M}_{I \times J \cdot K}(\mathbb{R})$, contains information from $J$ sensors at $K$ time instants and $I$ experimental trials. Consequently, each row vector represents for a specific experimental trial, the measurements from all the sensors at a particular time instant. Equivalently, each column vector represents measurements from one sensor at one particular time instant in the whole set of experimental trials.

The main objective of principal component analysis is to distinguish which dynamics are more relevant in the system, which are redundant and which can be considered as a noise (6). This objective is essentially accomplished by defining a new coordinate space to re-express the original one, by maximizing the variance and minimizing the correlation between variables in the new space. In other words, the objective is to find a linear transformation orthogonal matrix $\mathbf{P} \in \mathcal{M}_{J \cdot K \times J \cdot K}(\mathbb{R})$ that will be used to transform the original data matrix $\mathbf{X}$ into the form:

$$
\mathbf{T}=\mathbf{X P} \in \mathcal{M}_{I \times J \cdot K}(\mathbb{R}) .
$$

$\mathbf{P}$ is usually called the principal components of the data set or loading matrix and matrix $\mathbf{T}$ is the transformed or projected matrix onto the principal component space, also called score matrix. The columns of the principal components (columns of $\mathbf{P}$ ) are the eigenvectors of the covariance matrix $\mathbf{C}_{\mathbf{X}}$ organized according to its associated eigenvalue in descending order. In this way,

$$
\mathbf{C}_{\mathbf{X}} \mathbf{P}=\mathbf{P} \Lambda,
$$

where

$$
\mathbf{C}_{\mathbf{X}}=\frac{1}{J \cdot K-1} \mathbf{X}^{T} \mathbf{X} \in \mathcal{M}_{J \cdot K \times J \cdot K}(\mathbb{R}),
$$

and the diagonal terms of matrix $\Lambda$ are the eigenvalues $\lambda_{i}, i=1,2, \ldots, J \cdot K$.

Using all the $J \cdot K$ principal components, that is, in the full dimensional case, the orthogonality of $\mathbf{P}$ implies $\mathbf{P P}^{T}=\mathbf{I}$. Therefore, the projection can be inverted to recover the original data as $\mathbf{X}=\mathbf{T} \mathbf{P}^{T}$. However, PCA also seeks to reduce the dimensionality of the data set $\mathbf{X}$ by choosing only a reduced number, $\varrho<J \cdot K$, of principal components, that is, only the eigenvectors related to the $\varrho$ highest eigenvalues. In this way, given the reduced matrix $\widehat{\mathbf{P}} \in \mathcal{M}_{J \cdot K \times \varrho}(\mathbb{R})$, the score matrix is defined as

$$
\widehat{\mathbf{T}}=\mathbf{X} \widehat{\mathbf{P}} \in \mathcal{M}_{I \times \varrho}(\mathbb{R}) .
$$

Now, it is not possible to fully recover $\mathbf{X}$, although $\widehat{\mathbf{T}}$ can be projected back onto the original $J \cdot K$-dimensional space to obtain a reconstructed data matrix as follows:

$$
\widehat{\mathbf{X}}=\widehat{\mathbf{T}} \widehat{\mathbf{P}}^{T} \in \mathcal{M}_{I \times J \cdot K}(\mathbb{R}) .
$$

The difference between the original and the reconstructed data matrices ( $\mathbf{X}$ and $\widehat{\mathbf{X}}$ ), which describes the variability not represented in the projections, is defined as the residual error matrix $\mathbf{E}$ as follows:

$$
\begin{aligned}
\mathbf{E} & =\mathbf{X}-\widehat{\mathbf{X}} \\
& =\mathbf{X}-\widehat{\mathbf{T}} \widehat{\mathbf{P}}^{T} \\
& =\mathbf{X}-\mathbf{X} \widehat{\mathbf{P}} \widehat{\mathbf{P}}^{T}
\end{aligned}
$$

so

$$
\mathbf{E}=\mathbf{X}\left(\mathbf{I}-\widehat{\mathbf{P}} \widehat{\mathbf{P}}^{T}\right) \in \mathcal{M}_{I \times J \cdot K}(\mathbb{R})
$$

For the sake of simplicity, the caret is removed from the reduced matrices $(\widehat{\mathbf{T}}$ and $\widehat{\mathbf{P}})$ in the rest of the paper.

If equations (8) and (11) are analyzed in terms of experimental trials, we obtain:

$$
t_{i}^{T}=x_{i}^{T} \mathbf{P} \in \mathbb{R}^{\varrho}, i=1, \ldots, I,
$$

and

$$
e_{i}^{T}=x_{i}^{T}\left(\mathbf{I}-\mathbf{P P}^{T}\right) \in \mathbb{R}^{J \cdot K}, i=1, \ldots, I,
$$

where, $x_{i}^{T} \in \mathbb{R}^{J \cdot K}$ denotes the vector of the $i$ th experimental trial - that is, the $i$ th row of the original matrix $\mathbf{X}-; t_{i}^{T}$ is the vector of the projection of $x_{i}$ onto the first $\varrho$ principal components -that is, the $i$ th row of the score matrix $\mathbf{T}-;$ and, finally, $e_{i}^{T}$ is the residual error of the $i$ th experimental trial $-i$ th row of matrix $\mathbf{E}-$. 
In the literature, several methods can be found to calculate the principal components (PCs) -matrix $\mathbf{P}$ in equation (5)and the projections - matrix $\mathbf{T}$ in equation (5)-. Some of them are focused on the computation of the whole set of principal components, for instance, using the singular value decomposition (SVD) of the data matrix (36). Others strategies try to compute uniquely a reduced number of PCs, such as, for instance, the power method described initially by Hotelling (37) or the QL algorithm presented by Wilkinson (38). An alternative way of identifying the required PCs is to use the nonlinear iterative partial least square algorithm (NIPALS) (39), which is more efficient and accurate for large matrices, but slower than SVD. On the other hand, artificial neural networks (ANN) provide a way of extending PCA, including non linear generalizations (40).

\section{Damage indices (control chart)}

PCA can be used to detect abnormal behavior in a process or system. Two well-known statistics are commonly used to this aim: the $Q$-statistic (or square prediction error (SPE)statistic) and the Hotellings $T^{2}$-statistic (also named $D$ statistic). The first one is based on analyzing the residual error matrix $\mathbf{E}$ to represent the variability of the data projection in the residual subspace. The second index is based in analyzing the score matrix $\mathbf{T}$ to check the variability of the projected data in the new space of the principal components. These indices are based on the assumption (generally stemming from the central limit theorem) that the underlying process follows approximately a multivariate normal distribution where the first moment vector is zero.

$Q$-statistic. This index denotes the change of the events which are not explained by the model spanned by the principal components. In other words, it is a measure of the difference, or residual, between a sample and its projection onto the model. The $Q$-statistic of $i$ th experimental trial is defined as the sum of the squared residuals of each variable as follows:

$$
\begin{aligned}
Q_{i} & =\left\|e_{i}\right\|^{2}=e_{i}^{T} e_{i}=\sum_{\ell=1}^{J \cdot K} e_{i, \ell}^{2} \\
& =x_{i}^{T}\left(\mathbf{I}-\mathbf{P P}^{T}\right)\left(\mathbf{I}-\mathbf{P P}^{T}\right)^{T} x_{i} \\
& =x_{i}^{T}\left(\mathbf{I}-\mathbf{P} \mathbf{P}^{T}\right)\left(\mathbf{I}-\mathbf{P P}^{T}\right) x_{i} \\
& =x_{i}^{T}(\mathbf{I}-\mathbf{P} \mathbf{P}^{T}-\mathbf{P} \mathbf{P}^{T}+\mathbf{P} \underbrace{\mathbf{P}^{T} \mathbf{P}}_{\mathbf{I}_{\varrho}} \mathbf{P}^{T}) x_{i} \\
& =x_{i}^{T}\left(\mathbf{I}-\mathbf{P} \mathbf{P}^{T}-\mathbf{P} \mathbf{P}^{T}+\mathbf{P} \mathbf{P}^{T}\right)^{x_{i}} \\
& =x_{i}^{T}\left(\mathbf{I}-\mathbf{P P}^{T}\right) x_{i}
\end{aligned}
$$

where $e_{i, \ell} \in \mathbb{R}$ denotes the $\ell$ th element of the vector $e_{i}$, $\ell=1, \ldots, J \cdot K$.

$T^{2}$-statistic. In statistics, Hotelling's $T^{2}$-statistic is a generalization of Student's t-statistic that is used in multivariate hypothesis testing. It denotes the inner change of principal component model. $T^{2}$-statistic of the $i$ th experimental trial is defined by the averaged sum of its projection into the new space as follows:

$$
\begin{aligned}
T_{i}^{2} & =\sum_{r=1}^{\varrho} \frac{t_{i, r}^{2}}{\lambda_{r}}=t_{i}^{T} \boldsymbol{\Lambda}^{-1} t_{i} \\
& =x_{i}^{T}\left(\mathbf{P} \boldsymbol{\Lambda}^{-1} \mathbf{P}^{T}\right) x_{i} \in \mathbb{R}
\end{aligned}
$$

where $t_{i, r} \in \mathbb{R}$ denotes the $r$ th element of the vector $t_{i}$-the projection onto the $r$ th principal component or $r$ th score of the $i$ experimental trial-; and $\lambda_{r} \in \mathbb{R}$ is the $r$ th eigenvalue -see equation (6)-. $T^{2}$-statistic only detects variations in the plane of the first $\varrho$ principal components which are greater than what can be explained by the common-cause variations. In other words, the $T^{2}$-statistic is a measure of the variation in each sample within the PCA model.

Normally, $Q$-statistic is much more sensitive than $T^{2}$ statistic. This is because $Q$ is very small and therefore any minor change in the system characteristics will be observable. $T^{2}$ has great variance and therefore requires a great change in the system characteristic to be detectable (41). The concept of principal components is depicted in Figure 1. A three dimensional data set is shown, where the data lie primarily in a plane, thus the data is well described by a two PC's. The first PC aligns with the greatest variation in the data while the second PC aligns with the greatest amount of variation that is orthogonal to the first PC. Besides, examples damage indices for trials with unusual variations inside ( $T^{2}$-statistic) and outside of the model ( $Q$-statistic) are shown (42).

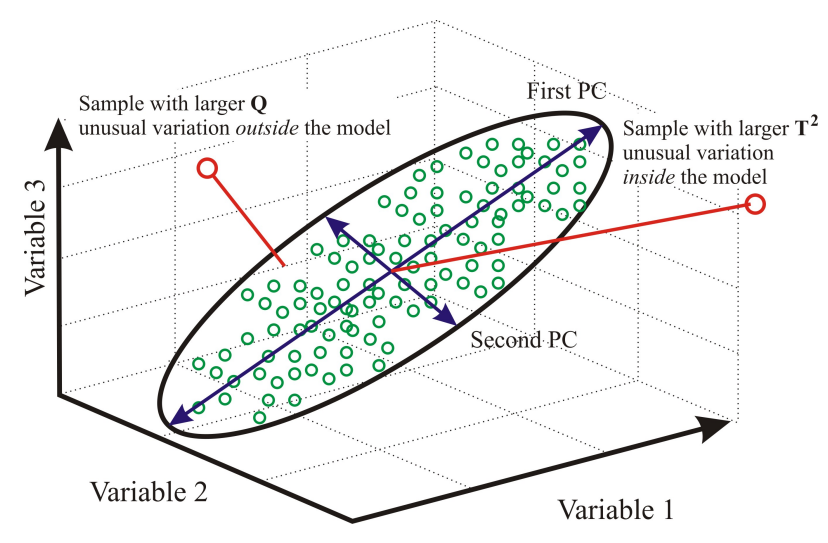

Figure 1. PCA model of three dimensional data set showing $T^{2}$-statistic and $Q$-statistic outliers (42).

\section{Generalized contribution plots in multiway principal component analysis}

The application of contribution plots for statistical process control (SPC) was introduced by Miller et al. to investigate the underlying PCA model when a fault detection exceeds its control limit (43). Scores and indices ( $Q$ and $T^{2}$-statistics) can determine whether a damage is present in the structure or not. However, they do not provide information about source, origin or cause. If an acceptable amount of historical data of all of the damages is available, classification methods can achieve convincing diagnosis results by assigning to the new measured data the damage belonging to the most similar class. Otherwise, developing and adequate classifier 
is inconceivable. An analysis of contribution can assign causes/localization of a damage. The main idea is to determine which sensor or sensors are measuring the outof-control signal, in other words, the contribution analysis indicates how each sensor is involved in the calculation of the damage index (44).

According to equations (14) and (15), the damage indicators are basically a sum of terms, each one associated with one variable (sensor-time in the case of unfolded matrix), called contributions. Once a damage has been detected because the indices ( $Q$ and $T^{2}$-statistics) take abnormal values, finding the elements of the signal $x_{i}$ which are mainly providing these values (highest contribution), means identifying the sensors, or more specifically, the wave propagation path that was more affected by the damage. This means that it can be inferred that the damage is located between the actuator and the identified sensor.

In general, the damage indicator indices of the $i$ th experimental trial can be expressed as:

$$
\mathbb{D}_{i}=\sum_{\ell=1}^{J \cdot K} \mathcal{C}\left(\mathbb{D}_{i}\right)_{\ell} \in \mathbb{R}
$$

where $\mathcal{C}\left(\mathbb{D}_{i}\right)_{\ell}$ is the contribution of the $\ell$ th variable to the index $\mathbb{D}$ of the $i$ th experimental trial. In this way, the vector of contributions of all variables to the index $\mathbb{D}_{i}$ is given by:

$$
\mathcal{C}\left(\mathbb{D}_{i}\right)^{T}=\left[\mathcal{C}\left(\mathbb{D}_{i}\right)_{1}, \mathcal{C}\left(\mathbb{D}_{i}\right)_{2}, \ldots, \mathcal{C}\left(\mathbb{D}_{i}\right)_{J \cdot K}\right] \in \mathbb{R}^{J \cdot K}
$$

\section{$Q$-statistic contribution}

Calculating the variable contributions to the $Q$-statistic is quite easy since this index is simply a squared prediction error summed over all the variables (equating equations (14) and (16)), the contribution of the variable $\ell$ to $Q$-statistic of the $i$ th experimental trial is given by:

$$
\begin{aligned}
\mathcal{C}\left(Q_{i}\right)_{\ell} & =e_{i, \ell}^{2}=\left[e_{i}^{T} \mathbf{e}_{\ell}\right]^{2} \\
& =\left[x_{i}^{T}\left(\mathbf{I}-\mathbf{P} \mathbf{P}^{T}\right) \mathbf{e}_{\ell}\right]^{2} \in \mathbb{R}
\end{aligned}
$$

where $\mathbf{e}_{\ell} \in \mathbb{R}^{J \cdot K}$ is the $\ell$-th vector of the canonical basis and it is used to indicate the variable $\ell$ whose contribution has to be computed. This is done by setting to 1 the position of the observation vector associated with the variable to compute its contribution, and the rest are set to 0 . An example of the variable vector for the first variable $\left(\mathbf{e}_{1}\right)$ of experimental trials with two sensors $(J=2)$ and two time instants by sensor $(K=2)$ would be:

$$
\mathbf{e}_{1}^{T}=\left[\begin{array}{llll}
1 & 0 & 0 & 0
\end{array}\right] \in \mathbb{R}^{2 \cdot 2} .
$$

This way, the vector of contributions of all variables is:

$$
\begin{aligned}
& \mathcal{C}\left(Q_{i}\right)=\left[\begin{array}{llll}
e_{i, 1}^{2} & e_{i, 2}^{2} & \cdots & e_{i, J \cdot K}^{2}
\end{array}\right] \\
& =\left[\left(x_{i}^{T}\left(\mathbf{I}-\mathbf{P P}^{T}\right) \mathbf{e}_{1}\right)^{2} \quad \ldots\right. \\
& \left.\cdots \quad\left(x_{i}^{T}\left(\mathbf{I}-\mathbf{P P}^{T}\right) \mathbf{e}_{J \cdot K}\right)^{2}\right] \in \mathbb{R}^{J \cdot K} .
\end{aligned}
$$

\section{$T^{2}$-statistic contribution}

$T^{2}$-statistic depends on the scores and those on the original variables. Consequently, it is possible to compute the influence of each original variable to this statistic. $T^{2}$ statistic can be decomposed as the square modulus of the $T^{2}$ contribution as follows:

$$
T_{i}^{2}=\left\|\mathcal{C}\left(T_{i}^{2}\right)\right\|^{2}=\mathcal{C}\left(T_{i}^{2}\right)^{T} \mathcal{C}\left(T_{i}^{2}\right) \in \mathbb{R} .
$$

In the other hand, equation (15) can re-expressed as:

$$
T_{i}^{2}=x_{i}^{T}\left(\mathbf{P}\left[\boldsymbol{\Lambda}^{-1}\right]^{1 / 2}\left[\boldsymbol{\Lambda}^{-1}\right]^{1 / 2} \mathbf{P}^{T}\right) x_{i} \in \mathbb{R}
$$

where the superscript $1 / 2$ represents the square root of a matrix, that is, $\left[\boldsymbol{\Lambda}^{-1}\right]^{1 / 2}$ is a matrix such that

$$
\left[\boldsymbol{\Lambda}^{-1}\right]^{1 / 2}\left[\boldsymbol{\Lambda}^{-1}\right]^{1 / 2}=\boldsymbol{\Lambda}^{-1}
$$

Relating the last two equations, it can be observed that the vector of contributions of all variables is determined by:

$$
\mathcal{C}\left(T_{i}^{2}\right)^{T}=x_{i}^{T} \mathbf{P}\left[\boldsymbol{\Lambda}^{-1}\right]^{1 / 2} \in \mathbb{R}^{J \cdot K}
$$

where $\mathcal{C}\left(T_{i}^{2}\right)$ is composed of the contribution of each variable to the statistic of the $i$ th experimental trial. Thus, it can be related to the individual contribution of each variable (equation (17)) as follows:

$$
\mathcal{C}\left(T_{i}^{2}\right)^{T}=\left[\mathcal{C}\left(T_{i}^{2}\right)_{1}, \mathcal{C}\left(T_{i}^{2}\right)_{2}, \ldots, \mathcal{C}\left(T_{i}^{2}\right)_{J \cdot K}\right] \in \mathbb{R}^{J \cdot K},
$$

where the contribution of the $\ell$ variables to the $T^{2}$-statistic of the $i$ th experimental trial is given by:

$$
\mathcal{C}\left(T_{i}^{2}\right)_{\ell}=\left(x_{i}^{T} \mathbf{P}\left[\boldsymbol{\Lambda}^{-1}\right]^{1 / 2} \mathbf{e}_{\ell}\right)^{2} \in \mathbb{R}
$$

\section{Sensor contribution}

The previous section explained the contribution of each variable to the given indicator once it has been detected the damage in one experimental trial. In any case, these contributions does not represent the contribution by sensor. The resulting vector $\mathcal{C}\left(\mathbb{D}_{i}\right) \in \mathbb{R}^{J \cdot K}$, where $J$ is the number of sensors and $K$ refers to the quantity of data-points by measurement (by sensor). To quantify the contribution of each sensor, several descriptive statistical measures were tested: kurtosis, skewness, mean, standard deviation and energy, being the last two the ones with better approximation. Therefore, from the contributions of the variables belonging to the same sensor, the energy and standard deviation of the set were calculated, in this way, only one number represent the contribution of all measurements from each sensors.

\section{Damage Localization Methodology}

\section{Overview}

The goal of this work is to determine the localization of damages in structures based on guided waves or vibrations. To achieve this goal, the structure is previously equipped with transductor devices that operate as actuator or sensors according with requirements and design of the experiments. 
The structure is subjected to a predefined number of experiments in which each trail experiment lies in the excitation of the specimen by means of one actuator and sensing the guided wave at different points of the structure (sensors). The gathered data from conducted experiments in the structure that we have previously considered as healthy or undamaged are used to train or build a baseline by means of PCA (PCA modeling). Once the structure needs to be inspected, it is subjected to the same procedure or experimentation. Data is projected onto the PCA model and the damage indices are calculated. Finally, an analysis of the contribution of each sensor to each damage indices is performed. The basis of the methodology proposed in this work lies in the hypothesis that the damage produces a change in the damage indicators (detection), and besides, this change is more affected by data from the sensor located in the direct route with the actuator. However, other sensors are also affected and this contribution should be analyzed. In the following sections, a detailed explanation of the whole methodology is presented.

\section{PCA modeling}

Let us consider the structure in a fully healthy state where we perform a set of experiments using a multiactuator system. For each actuation phase (exciting actuator 1 and sensing in all sensors, exciting actuator 2 and sensing in all sensors, and so on) the recorded signals become part of the matrix $\mathbf{X}_{\text {und }}$ organized as in equation (3) and scaled as explained in (6). PCA modeling essentially consists of calculating the projection matrix $\mathbf{P}$ for each phase (equation (5)), which offers a better and dimensionally reduced representation of the original data $\mathbf{X}_{\text {und }}$. Matrix $\mathbf{P}$, renamed $\mathbf{P}_{\mathbf{m}}$, will be considered as the model of the undamaged structure, besides the damage statistics by each trial $Q_{u n d}$ and $T_{u n d}^{2}$ from equations (14) and (15) will be used in the diagnosis as illustrated in Figure 2.

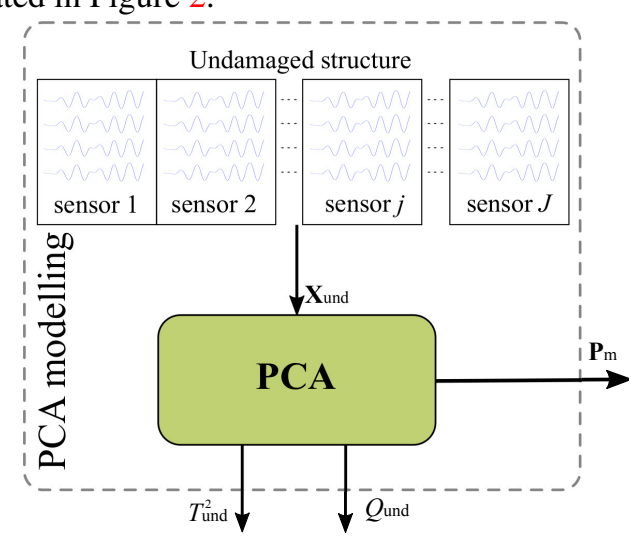

Figure 2. PCA modeling

\section{Damage detection}

The current structure to diagnose is subjected to a predefined number of experiments and a new data matrix $\mathbf{X}_{\mathbf{c}}$ is constructed with the measured data. The number of experiments can be as many as wanted, but the number of sensors and collected samples (data-points) must be the same as it was used in the modeling stage; that is, the number of columns of $\mathbf{X}_{\mathbf{c}}$ must agree with that of $\mathbf{X}_{\mathbf{h}}$. This matrix $\mathbf{X}_{\mathbf{c}}$ is projected onto the baseline PCA model (equation (5)). Damage statistics by each trial $Q_{c}$ and $T_{c}^{2}$ from equations (14) and (15) are calculated to obtain the damage indicator (see Figure 3).

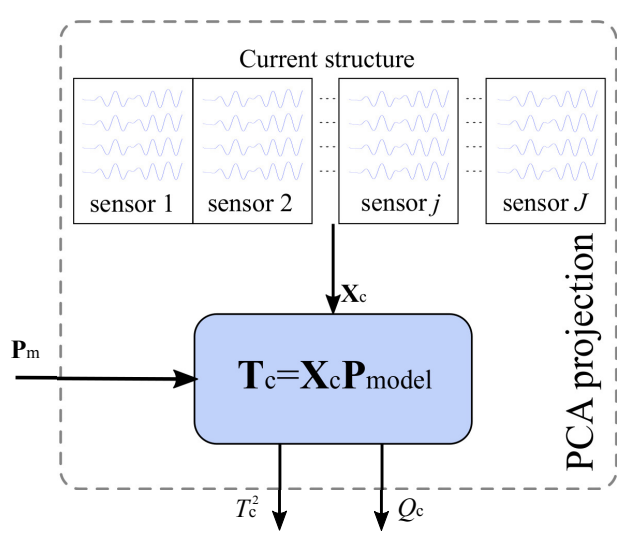

Figure 3. PCA projection

To detect whether the current structure has some damage or not, its damage indices must be compared with the obtained from the undamaged structures (see Figure 4). Sometimes, a visual comparison is enough to detect the damage (6). In other cases, however, it is necessary to complement the detection by means of a classifier or hypothesis testing (8).

\section{Contribution analysis}

Once it is determined that the current structure has a damage, the methodology for localizing the damage is carried out. Here it is analyzed the contribution of each sensor to the damage indices which has detected the damage. As explained previously, these contributions are calculated by means of equations (20) or (26), the energy and standard deviation of the contributions by sensor were calculated, and finally they are plotted if it is necessary. It can appreciated that the contribution of this sensor which has been used as actuator is null (no contribution to the damage indices) as shown in Figure 5.

\section{Damage localization}

As mentioned at the beginning of this section, the procedure is implemented by each actuation phase of the multi-actuator system. To localize the damage, we combine the result of all the actuation phases, by each actuation phase the contribution is calculated. Based on the hypothesis that the damage is located in the path between the actuator and the sensor with largest contribution, this path is marked as the most probably region with damage. However, other sensors have also contributions, therefore, there is some probability that damage is located in these routes. In this way, a map or grid of the surface of the structure is constructed indicating all actuators/sensors and all possible routes. All routes are weighted according to the contribution in each phase. Finally, the superposition of these routes give us the region with more probability of localization as shown in Figure 6. 


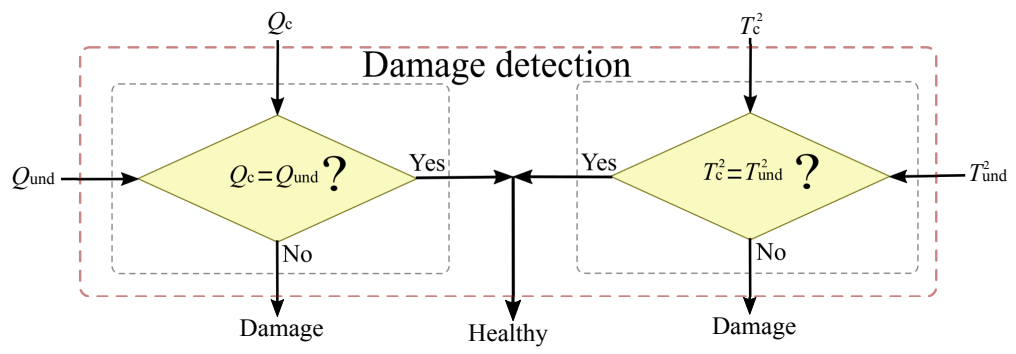

Figure 4. Damage detection

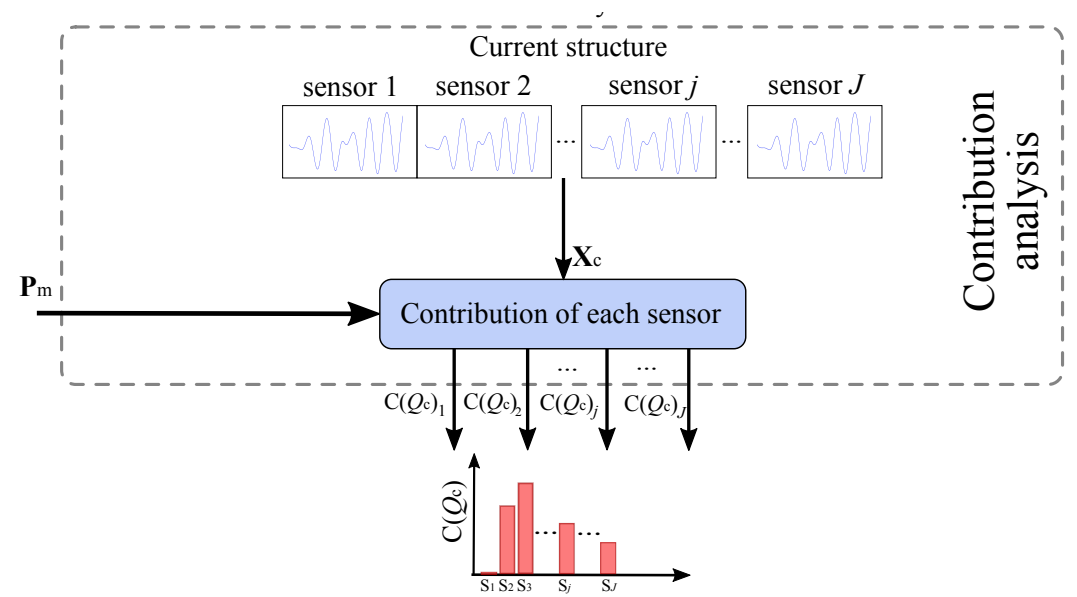

Figure 5. Contribution analysis

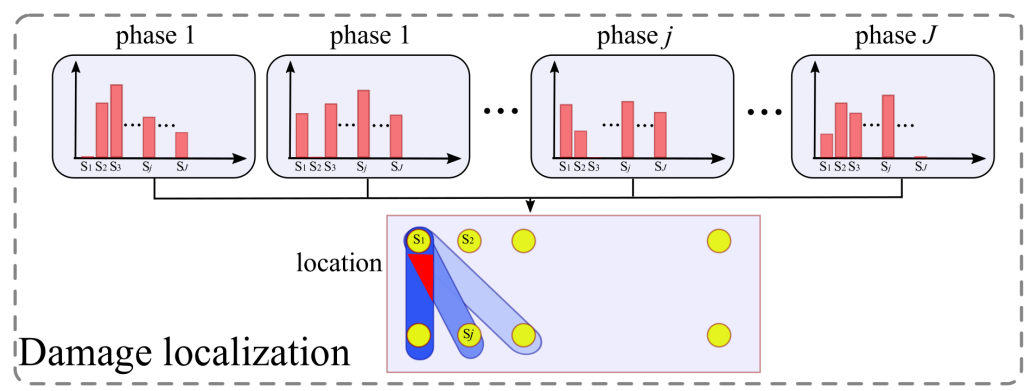

Figure 6. Damage localization

\section{Experimental Setup}

The paper is focused on the methodology for signal processing to damage localization based on guided waves, it is not focused on the structure itself. To illustrate and demonstrate the feasibility of the approach, a fan stage blade of a commercial aircraft turbine is employed in this work. It has been previously used by the authors applying several extensions of Statistical Models for instance, (6). Unfortunately, due to the origin of the specimen (donation from a manufacturing company for research purposes), little is known about the material specifications, and design parameters constituting the structure. However, it could be determined that it is manufactured by a homogeneous material with a similar density that titanium (about 4.5 $\left.\mathrm{g} / \mathrm{cm}^{3}\right)$. Its size is $50 \times 15 \times 1 \mathrm{cms}$ approximately and, around $4 \mathrm{~kg}$ weight. This structure besides the stringers, has some flaws, however since the proposed method is based on pattern recognition comparing the current response with a baseline, this imperfect structure can be considered as healthy

The blade was suspended by two elastic ropes and eight PZT sensors are distributed over the surface as can be seen from Figure 7. In each experiment, one of the PZT's is chosen as actuator and the other seven as sensors. The actuator was excited by a burst signal of three peaks and $200 \mathrm{kHz}$ of central frequency. Next, another PZT is used as actuator and the rest as sensors, and so on.

Since it was not possible to drill or saw the blade (seeded damage), no permanent damages were induced to the structure, two masses were added at 15 different locations as shown in Figure 8. Results are shown using the smallest mass, only few tens of grams. It means that the ratio between the mass of the damage and the mass of the structure is around $0.01(1 \%)$. 


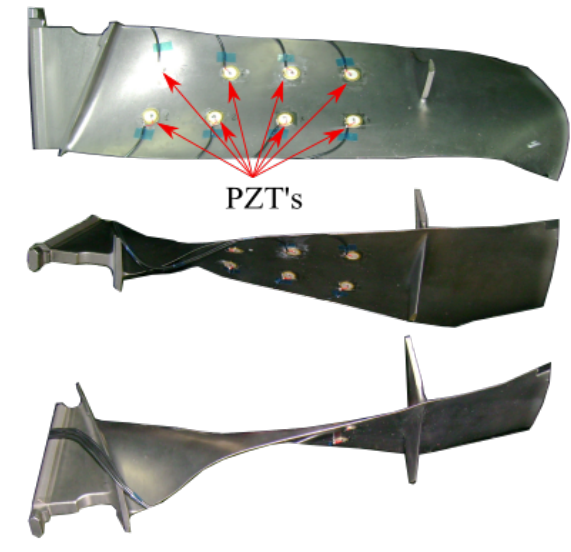

Figure 7. Specimen: blade of a commercial aircraft turbine and localization of PZT's.

210 experiments were performed and recorded: 60 with the undamaged structure, and 150 with the damaged structure (10 per damage). Each experiment has 16000 time data points for each one of the seven sensors. 50 experimental trials of the first data set undamaged structure was used for modeling the PCA model. The rest from the undamaged structure and the second data set damaged structure was used for testing the approach. Finally, all the gathered data are collected and organized as specified in equation (3).

\section{Analysis of the results and discussion}

Since the paper is focused on damage localization, results presented in this section does not include the ones concerning to detection of the damage. We will be focused on presenting the resulting contributions and the final localization. As was previously explained, the structure was excited in one PZT with a burst signal of 3 peaks with central frequency of 200 $\mathrm{kHz}$ as shown above of Figure 9. As illustrative example, we have selected the structure when the mass is added in the position 3 . The recorded guided wave in sensors 2,3,4 and 5 are also shown in Figure 9. The sample rate was $48 \mathrm{MHz}$, obtaining a total of 16000 data-points by signal. Its is very clear how arrive the guide wave to all sensors and how much they are influenced by the direct wave and all the reflections due the contour and stringers of the blade.

Once the data is organized, the PCA model is built and the projection of the data from current structure is conducted, the damage indices are compared with the indices from the pristine structure. In Figure 10, it can be seen the projection of all conditions of the structure. It is very clear by a simple eye inspection that all damages are very well separated from the healthy cases, moreover, some of them are separated from the others (distinction between damages). These results have been widely analyzed in previous works from the authors.

Since the damage indices determine that a damage is present in the blade, the contributions of the variables to these indices are calculated. Continuing with the illustrative example, contribution of all variables to the $Q$-statistic are depicted in Figure 11. Remind that each variable is represented by each column of the matrix $\mathbf{X}$ in equation (3), so the resulting contributions is a vector of 112000 elements (16000 data-points $\times 7$ sensors).
To quantify the contribution of each sensor, the energy and the standard deviation of the signal by sensor are calculated. In Figure 12 it can be seen the energy of the contributions by sensors in the mentioned example. It is important to notice that the contribution of PZT 1 is null since this PZT was used as actuator. On the other hand, PZT 7 presents the greatest contribution to the index $Q$. This means, the damage that produces the growth of the index and subsequently reveals the alert, can be located in the route between PZT 1 and PZT 7. However, PZT 6 also contributes; and PZT 4, and so on.

Since all this process has to be conducted for all the actuation phases, we can build a map of the structure indicating the position of all PZT's and the weight of each route between PZT's is determined by the sum of the contributions by each actuation phase. By using a typical method of interpolation and construction of a surface meshgrid, we can assign the final contribution to the routes and most probably localization of the damage can be easily visualized. Figures from 13 to 21 shows the final localization of damages 1 to 9 as a level curve over the photography of the real structure with the adhered mass, the rest of damages are not showed but they are commented.

From these results, it can be appreciated the possible localizing region of the proposed added mass that we have considered as damages. Damage 1 (hereinafter referred to as D1) is located outside of the array of sensors, the mass is not laid between any direct route between PZT's. However, due to reflections of the guided wave in the boundary of the specimen, an acceptable estimation of the localization is achieved.

Some damages are perfectly located (e.g. 2, 5, 7 and 9). In other cases, the methodology proposes two localization regions, being one of them the right one (e.g. 3, 4 and 6). D8 (Figure 20) is curious because the approach does not propose one or two regions, but it proposes four. These four regions have their probabilities and the highest one in that belonging to the left (close to PZT 1) being the correct region where the damage is located.

Finally, the other damages were detected but not located (not shown in this paper). One specific example about detection (even separation between damages) but not localization can be observed in Figure 10. It is clear that D9 (yellow diamond) and D14 (green leftward-pointing triangle) are so close, therefore they are detected and distinguished between them. However, contrary to D9, D14 is not located. As perceived in Figure 8, these both damages are symmetrically located, this can indicate us that symmetry in the array of sensors and damages can affect the accuracy of the approach.

Similarly, it can be seen that D8, D10, D13 and D15 are also symmetrically laid. The mentioned damages (cyan circle, red upward-pointing triangle, cyan right-pointing triangle and blue downward-pointing triangle respectively) are very close in Figure 10, all of them are detected but only D8 is correctly located. Again, this symmetry could explain the looseness for determining the possible region where the damage is located. Finally, D6 and D11 (blue circle and yellow star) are also symmetrically laid and correctly detected (but not distinguished between them at all), however D11, contrary to D9, is not located. 


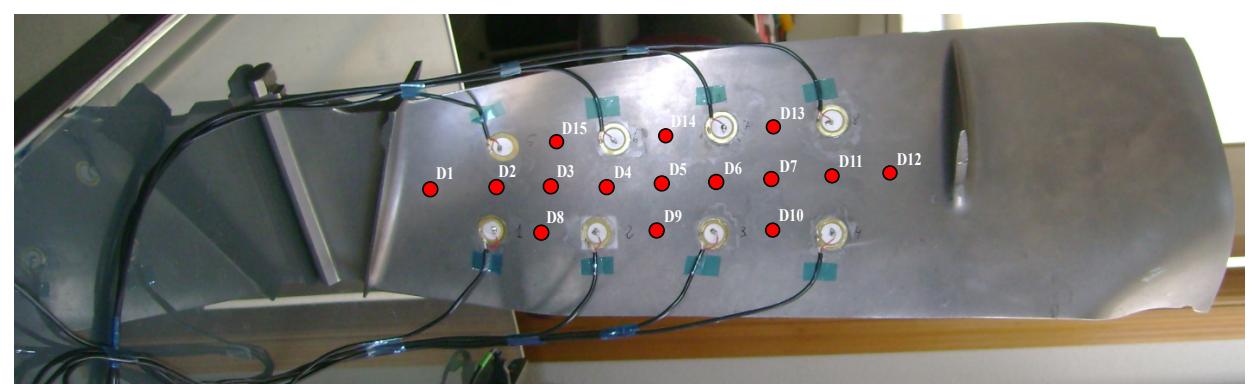

Figure 8. Adhered mass to simulate damages and its localizations.
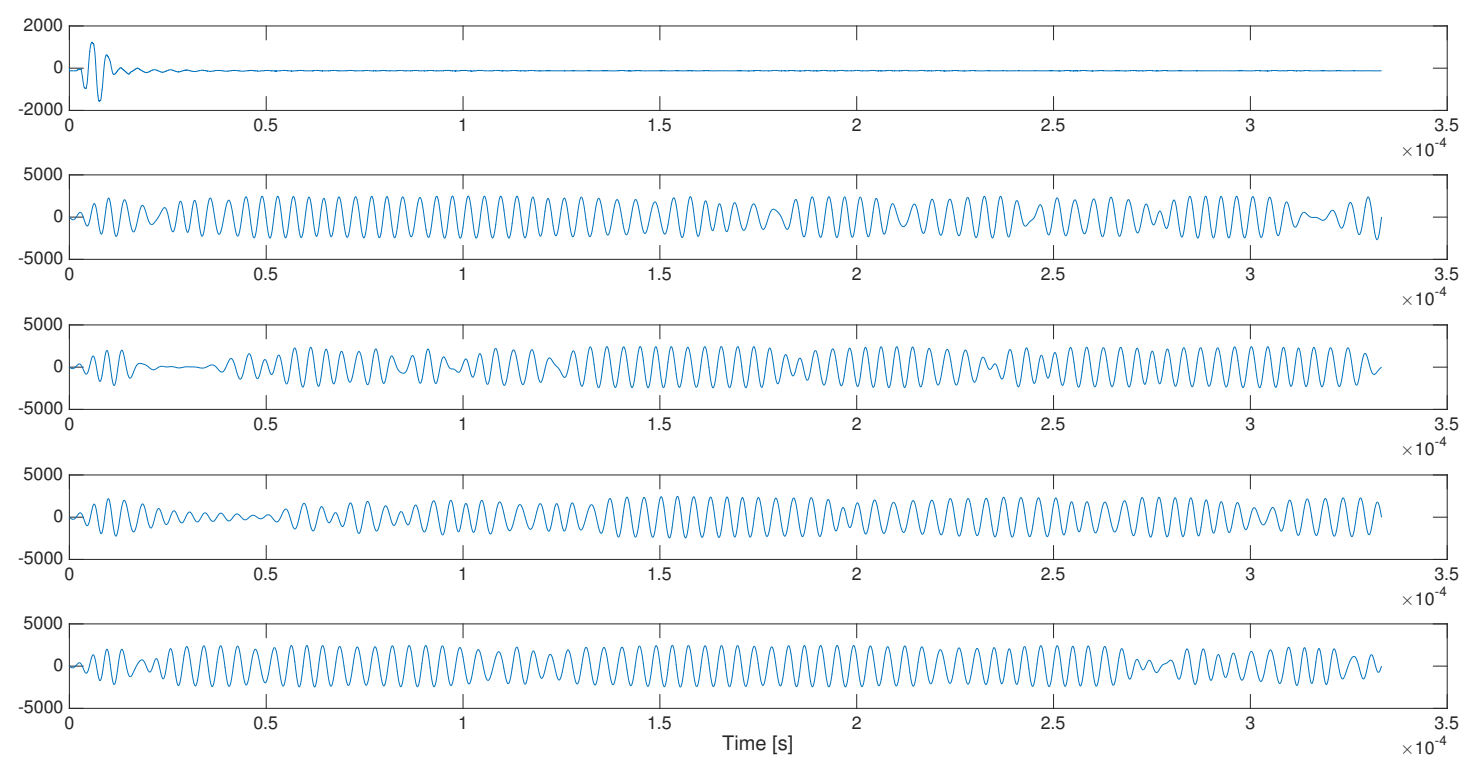

Figure 9. Example of recorded signals: Actuation in PZT 1 and sensing in PZT's 2,3,4 and 5 respectively when the specimen is subjected to D3

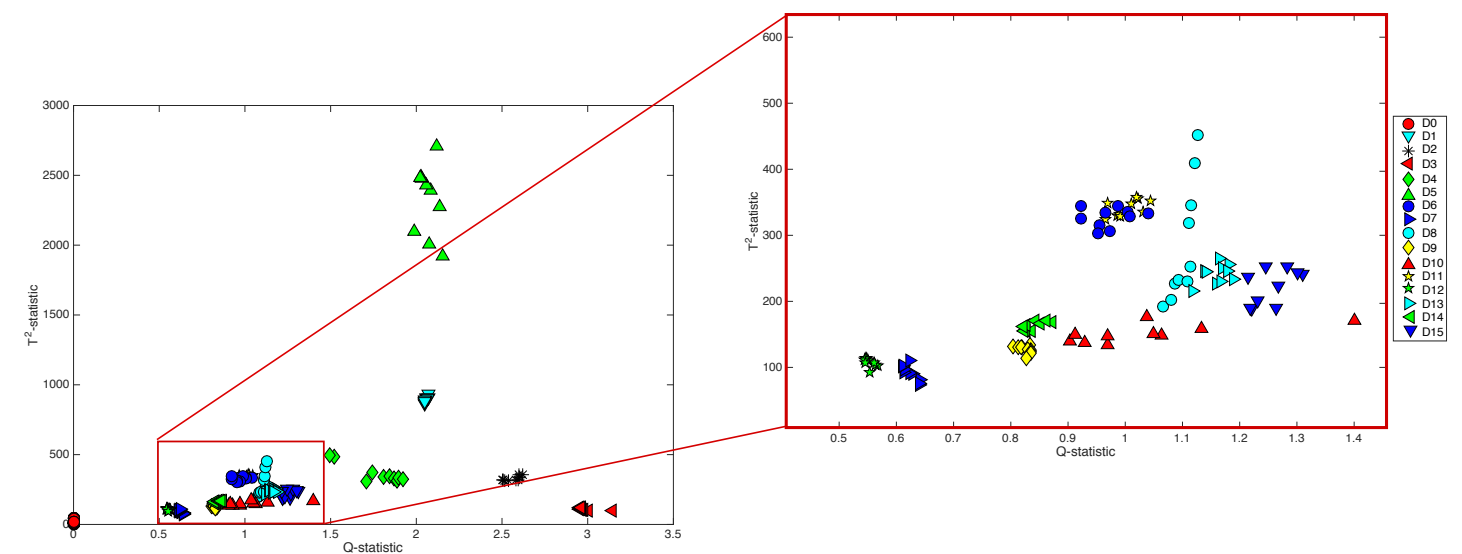

Figure 10. Damage indices of all experimental records.

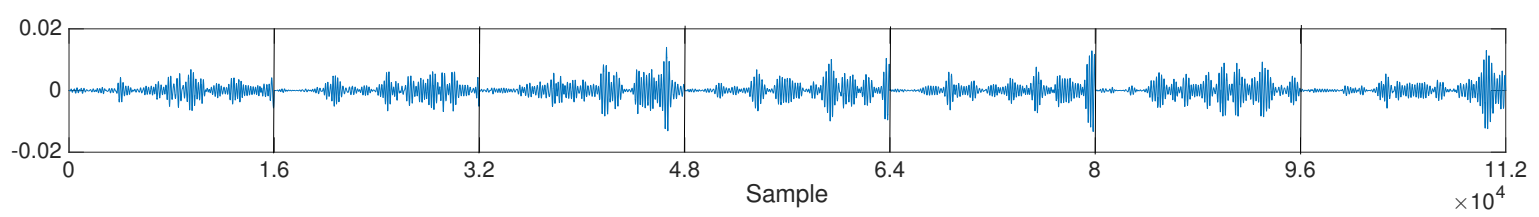

Figure 11. Example of contributions of each variable to $Q$-statistic: Actuation in PZT 1 when the specimen is subjected to D3 


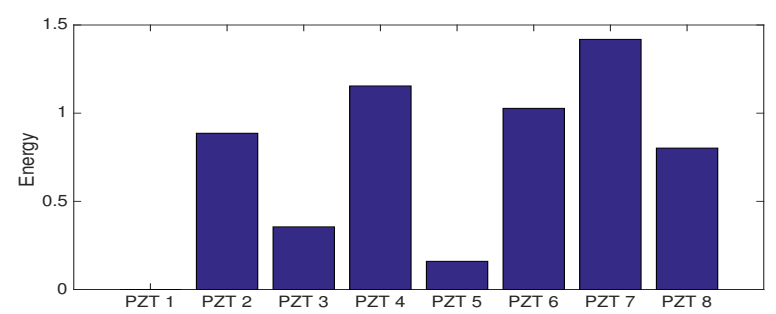

Figure 12. Example of energy of contributions of each sensor to $Q$-statistic.

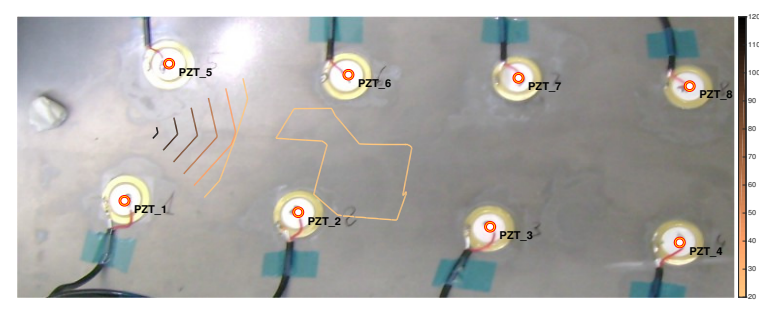

Figure 13. Localization of damage 1

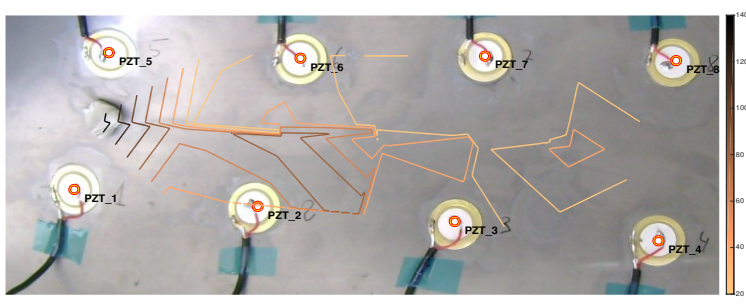

Figure 14. Localization of damage 2

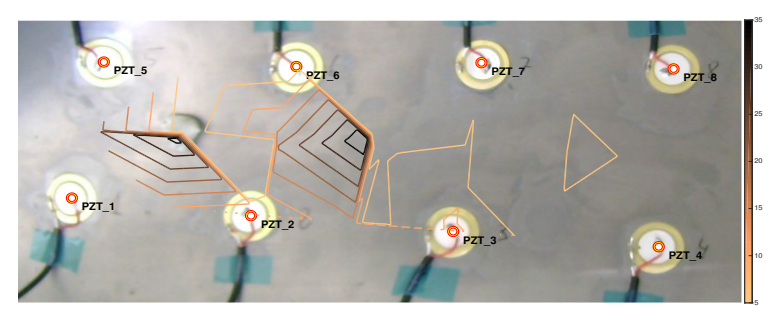

Figure 15. Localization of damage 3

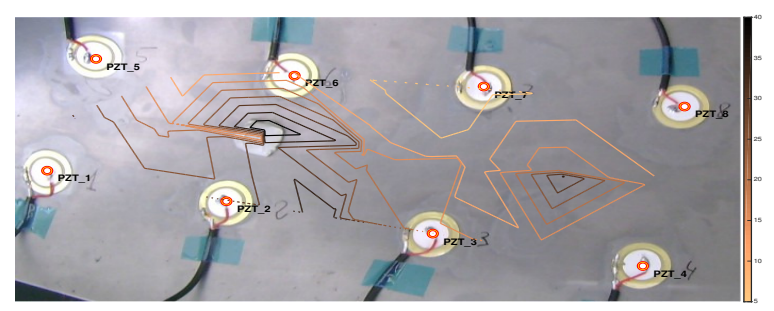

Figure 16. Localization of damage 4

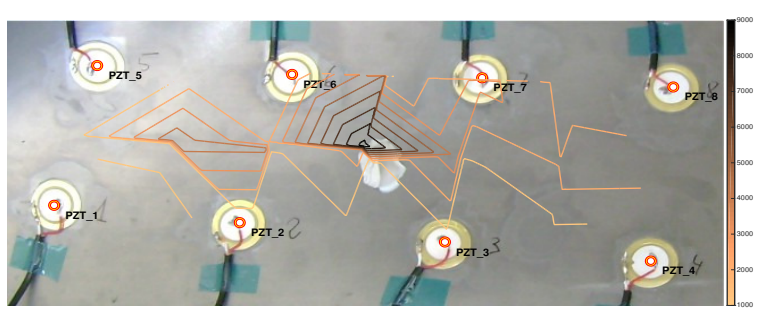

Figure 17. Localization of damage 5

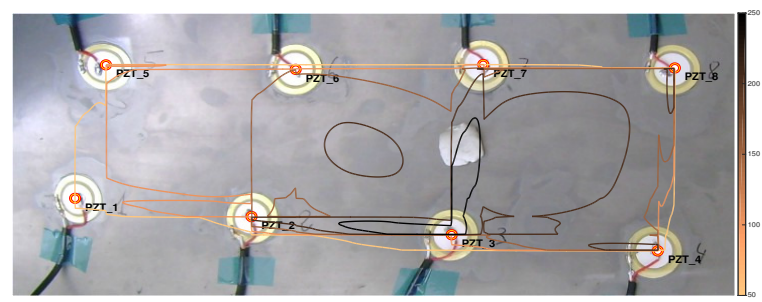

Figure 18. Localization of damage 6

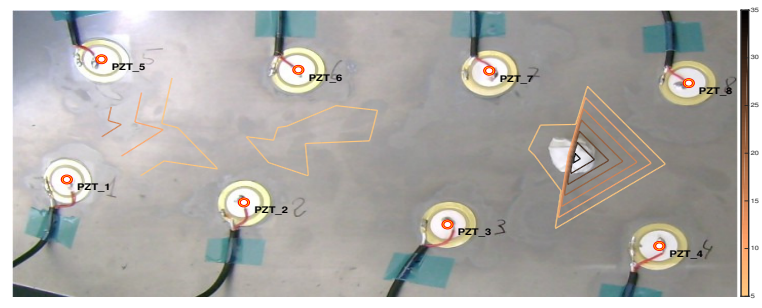

Figure 19. Localization of damage 7

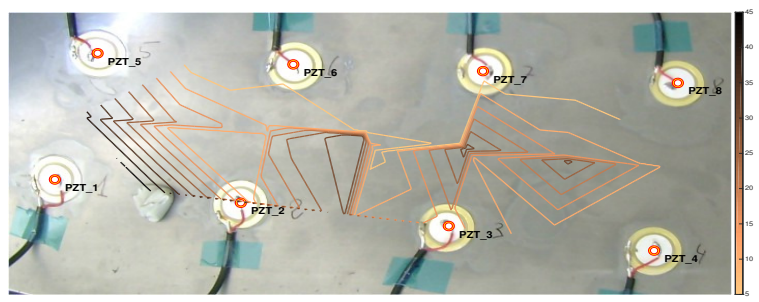

Figure 20. Localization of damage 8

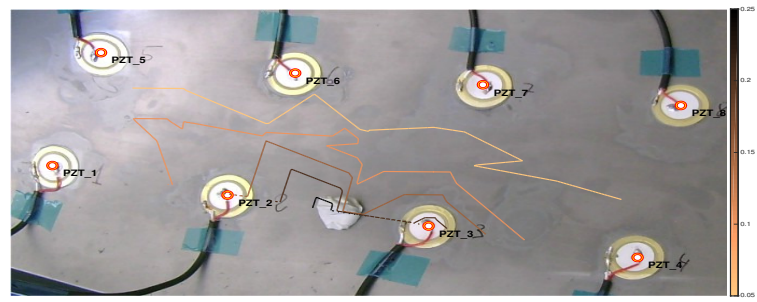

Figure 21. Localization of damage 9 


\section{Conclusions}

In this paper, a novel methodology for damage localization is introduced. The approach is based on the following elements: (i) the structure to diagnose is equipped with a multi-actuator system, this means that itself has the ability of exciting the specimen and measuring its response at different points; (ii) actuators/sensors are configured in pitch-catch mode; (iii) once the specimen is excited by one of its actuators, the damage affects the normal travel of the guided wave, and this change is mainly detected by sensors in the direct path with the excitation point; and (iv) the multivariable analysis based on principal component analysis (PCA) of all recorded signals determines whether the damage is present. But the analysis of the contribution of each sensor to this index which gives the alarm, can determine its localization.

The main advantages of the proposed methodology comparing with other existing studies are: (i) only the measurements from PZT sensors distributed over the structure surface are necessary. (ii) PCA is the unique tool implemented. This methodology is not completed with other techniques. With a simple analysis about how much each sensor measurements have contributed in each principal component, the methodology provides a possible region where the damage can be localized. In a past work, the authors have implemented the partial least square algorithm (PLS) (referenced in the introduction section) for impact localization. PLS could quickly summarized as a supervised PCA in which, for the training step, the previous knowledge about the coordinates $(x, y)$ of the impact are necessary and essential. Which brings the next advantage: (iii) in the present work, no previous information of the damage location is required.

The proposed methodology has been applied and validated on an aircraft turbine blade. The results indicate that the presented methodology is able to accurately locate damages, analyzing the record signals from all actuation phases, giving a unique and reliable region. As drawback, it can be mentioned that in some cases the methodology does not provide only one possible region but if these possible regions are examined and analyzed, the damage can be located. On the other hand, in some cases due to the symmetry of the location of the added mass, some damages are detected but not correctly localized.

In the literature, more damage indicators based on PCA or another similar multivariable technique can be found. On the other hand, it can be studied more methods of contributions analysis, this is, another mathematical approaches that can determine how each variable/sensor is responsible of the damage detection. As future work, authors intend to analyze all these indicators and contribution methods to provide a complete study of their advantages/drawbacks and applications.

\section{Acknowledgements}

This work has been partially funded by the Spanish Ministry of Economy and Competitiveness through the projects DPI2014$58427-\mathrm{C} 2-1-\mathrm{R}$ and by the Catalonia Government through the project 2014SGR859. The authors are grateful also to professor Alfredo Güemes who has collaborated with the collection of data and contributed with valuable information.

\section{References}

[1] Farrar CR and Worden K. An introduction to structural health monitoring. Philos. Transactions of the Royal Society of London A: Mathematical, Physical and Engineering Sciences 2007; 365(1851): 303-315. DOI:10.1098/rsta.2006.1928.

[2] $\mathrm{Su} \mathrm{Z}$ and Ye L. Identification of Damage Using Lamb Waves. Lecture Notes in Applied and Computational Mechanics. ISBN 978-1-84882-783-7. DOI:10.1007/ 978-1-84882-784-4.

[3] Wold S, Geladi P, Esbensen K et al. Multiway pincipal component and PLS analysis. Journal of Chemometrics 1987; 1: 41-56.

[4] Nomikos $\mathrm{P}$ and MacGregor JF. Monitoring batch processes using multiway principal component analysis. AIChE Journal 1994; 40(8): 1361-1375.

[5] Kourti T. Process analysis and abnormal situation detection: From theory to practice. IEEE Control Systems Magazine 2002; 22(5): 10-25.

[6] Mujica L, Rodellar J, Fernández A et al. Q-statistic and T2-statistic PCA-based measures for damage assessment in structures. Structural Health Monitoring 2011; 10(5): 539553.

[7] Ruiz M, Mujica LE, Berjaga $X$ et al. Partial least square/projection to latent structures (pls) regression to estimate impact localization in structures. Smart Materials and Structures 2013; 22(2): 025028.

[8] Mujica LE, Ruiz M, Pozo F et al. A structural damage detection indicator based on principal component analysis and statistical hypothesis testing. Smart Materials and Structures 2014; 23(2): 025014.

[9] Mujica LE, Rodellar J and Vehí J. A Review of Impact Damage Detection in Structures using Strain Data. International Journal of COMADEM 2010; 13(1): 3-18.

[10] Park K, Darsono D and Torbol M. Damage localization using modal submatrix scaling factor and neural network. In Proceedings of 8th European Workshop On Structural Health Monitoring (EWSHM 2016). Bilbao, Spain.

[11] Limongelli MP and Morassi A. Experimental verification of the interpolation method on a real damaged bridge. Journal of Physics: Conference Series 2015; 628(1): 012045.

[12] Wang X, Tse PW, Mechefske CK et al. Experimental investigation of reflection in guided wave-based inspection for the characterization of pipeline defects. $\{N D T\} \& E$ International 2010; 43(4): 365 - 374. DOI:http://dx.doi.org/ 10.1016/j.ndteint.2010.01.002.

[13] Jiao JP, Drinkwater BW, Neild SA et al. Low-frequency vibration modulation of guided waves to image nonlinear scatterers for structural health monitoring. Smart Materials and Structures 2009; 18(6): 065006.

[14] Michaels JE and Michaels TE. Guided wave signal processing and image fusion for in situ damage localization in plates. Wave Motion 2007; 44(6): 482 - 492. DOI:http://dx.doi.org/ 10.1016/j.wavemoti.2007.02.008.

Special Issue of Selected Papers Presented at the International Symposium on Mechanical Waves in Solids.

[15] Li B, Liu Y, Gong K et al. Damage localization in composite laminates based on a quantitative expression of anisotropic wavefront. Smart Materials and Structures 2013; 22(6): 065005. 
[16] Dai D and He Q. Structure damage localization with ultrasonic guided waves based on a time-frequency method. Signal Processing 2014; 96, Part A(0): 21 - 28. DOI:http: //dx.doi.org/10.1016/j.sigpro.2013.05.025. Time-frequency methods for condition based maintenance and modal analysis.

[17] Tua PS, Quek ST and Wang Q. Detection of cracks in plates using piezo-actuated lamb waves. Smart Materials and Structures 2004; 13(4): 643.

[18] Wang CS, Wu F and Chang FK. Structural health monitoring from fiber-reinforced composites to steel-reinforced concrete. Smart Materials and Structures 2001; 10(3): 548.

[19] Ostachowicz W, Kudela P, Malinowski P et al. Damage localisation in plate-like structures based on $\{\mathrm{PZT}\}$ sensors. Mechanical Systems and Signal Processing 2009; 23(6): 1805 - 1829. DOI:http://dx.doi.org/10.1016/j.ymssp.2008.10.011. Special Issue: Inverse Problems.

[20] Flynn EB, Todd MD, Wilcox PD et al. Maximum-likelihood estimation of damage location in guided-wave structural health monitoring. Proceedings of the Royal Society of London A: Mathematical, Physical and Engineering Sciences 2011; 467(2133): 2575-2596. DOI:10.1098/rspa.2011.0095.

[21] Moll J. Guided Wave-based Damage Localization in Isotropic Structures with Smoothly Varying Thickness. In Proceedings of 8th European Workshop On Structural Health Monitoring (EWSHM 2016). Bilbao, Spain.

[22] Moll J, Schulte RT, Hartmann B et al. Multi-site damage localization in anisotropic plate-like structures using an active guided wave structural health monitoring system. Smart Materials and Structures 2010; 19(4): 045022.

[23] Moll J, Torres-Arredondo MA and Fritzen CP. Computational aspects of guided wave based damage localization algorithms in flat anisotropic structures. Smart Structures and Systems 2012; 10(3): 229-251.

[24] Levine RM and Michaels JE. Block-sparse reconstruction and imaging for lamb wave structural health monitoring. IEEE Transactions on Ultrasonics, Ferroelectrics, and Frequency Control 2014; 61(6): 1006-1015. DOI:10.1109/TUFFC.2014. 2996.

[25] Harley JB and Moura JM. Data-driven matched field processing for lamb wave structural health monitoring. The Journal of the Acoustical Society of America 2014; 135(3): 1231-1244.

[26] Bhuyan MDH, D?hler M and Mevel L. Statistical Damage Localization with Stochastic Load Vectors Using Multiple Mode Sets. In Proceedings of 8th European Workshop On Structural Health Monitoring (EWSHM 2016). Bilbao, Spain.

[27] Johansen J, Ulriksen M and Damkilde L. DELORES - A System for Detection and Localization of Structural Damages. In Proceedings of 8th European Workshop On Structural Health Monitoring (EWSHM 2016). Bilbao, Spain.

[28] Kopsaftopoulos F and Fassois S. A functional model based statistical time series method for vibration based damage detection, localization, and magnitude estimation. Mechanical Systems and Signal Processing 2013; 39(1): 143 - 161. DOI: http://dx.doi.org/10.1016/j.ymssp.2012.08.023.

[29] Sakaris CS, Sakellariou JS and Fassois SD. Vibration-based damage precise localization in three-dimensional structures: Single versus multiple response measurements. Structural Health Monitoring 2015; 14(3): 300-314. DOI:10.1177/ 1475921714568407.
[30] Eremin A, Glushkov E, Glushkova N et al. Analytically based time-reversal technique for damage localization and characterization in laminate composite structures. In Proceedings of 8th European Workshop On Structural Health Monitoring (EWSHM 2016). Bilbao, Spain.

[31] Liu K, Ma S, Wu Z et al. A novel probability-based diagnostic imaging with weight compensation for damage localization using guided waves. Structural Health Monitoring 2016; 15(2): 162-173. DOI:10.1177/1475921715627491.

[32] Kosanovich K, Piovoso M, Dahl K et al. (eds.). Multiway PCA applied to an industrial batch process. American Control Conference, 1994.

[33] Duchesne C, Kourti T and MacGregor JF (eds.). Multivariate Monitoring of startups, restarts and grade transitions using projection methods. 0-7803-789, American Control Conference, 2003.

[34] Westerhuis JA, Kourti T and MacGregor JF. Comparing alternative approaches for multivariate statistical analysis of batch process data. Journal of Chemometrics 1999; 13: 397413.

[35] Wold S, Geladi P, Esbensen K et al. Multiway pincipal component and pls analysis. Journal of Chemometrics 1987; 1: 41-56.

[36] Chambers JM. Computational methods for data analysis / John M. Chambers. Wiley New York, 1977. ISBN 0471027723 .

[37] Hotelling H. Analysis of a complex of statistical variables with principal components. Journal of Educational Psychology 1933; 24: 417-441.

[38] Wilkinson JH. The Algebraic Eigenvalue Problem. Monographs on Numerical Analysis. Oxford Science Publications, Oxford: The Clarendon Press, Oxford University Press, 1988. ISBN 0-19-853418-3. Paperback reprint of original edition, published in 1965 by Clarendon Press, Oxford.

[39] Wold H. Estimation of principal components and related models by iterative least squares. In Krishnaiah KR (ed.) Multivariate Analysis. New York: Academic Press, 1966. pp. 391-420.

[40] Jolliffe IT. Principal Component Analysis. Second edi ed. Springer Series is Statistics, Springer-Verlag, 2002.

[41] Qin SJ. Statistical process monitoring: Basics and beyond. Journal of Chemometrics 2003; 17(8-9): 480-502.

[42] Wise BM, Gallagher NB, Watts S et al. A comparison of pca, multiway pca, trilinear decomposition and parallel factor analysis for fault detection in a semiconductor etch process. Journal of Chemometrics 1999; 13: 379-396.

[43] Miller P, Swanson R and Heckler C. Contribution plots: a missing link in multivariate quality control. Applied Mathematics and Computer Science 1998; 8(4): 775-792.

[44] Kourti T and MacGregor JF. Multivariate SPC Methods for Process and Product Monitoring. Journal of Quality Technology 1996; 28: 409-428. 